\title{
A HUREWICZ-TYPE THEOREM FOR APPROXIMATE FIBRATIONS
}

\author{
D. S. CORAM AND P. F. DUVALL, JR. ${ }^{1}$
}

\begin{abstract}
This paper concerns conditions on point inverses which insure that a mapping between locally compact, separable, metric ANR's is an approximate fibration. Roughly a mapping is said to be $\pi_{i}$-movable [respectively, $\boldsymbol{H}_{i}$-movable] provided that nearby fibers include isomorphically into mutual neighborhoods on $\pi_{i}$ [resp. $H_{i}$ ]. An earlier result along this line is that $\pi_{i}$-movability for all $i$ implies that a mapping is an approximate fibration. The main result here is that for a $U V^{1}$ mapping, $\pi_{i}$-movability for $i<k-1$ plus $H_{k}$ - and $H_{k+1}$-movability imply $\pi_{k}$-movability of the mapping. Hence a $U V^{1}$ mapping which is $H_{i}$-movable for all $i$ is an approximate fibration. Also, if a $U V^{1}$ mapping is $\pi_{i}$-movable for $i<k$ and $k$ is at least as large as the fundamental dimension of any point inverse, then it is an approximate fibration. Finally, a $U V^{1}$ mapping $f: M^{m} \rightarrow N^{n}$ between manifolds is an approximate fibration provided that $f$ is $\pi_{i}$-movable for all $i<\max \{m-n$, $\left.\frac{1}{2}(m-1)\right\}$.
\end{abstract}

1. Introduction and statement of results. Given a proper surjective mapping $p$ : $E \rightarrow B$ between locally compact, separable ANR's, we are interested in conditions on the point inverses which insure that $p$ is an approximate fibration (definition below). Earlier results in this direction involve " $U V$ " conditions on point inverses. The mapping $p: E \rightarrow B$ is said to be a $k$-uv [resp., $k-U V$ ] mapping provided that $p$ is proper and surjective and for every $b$ in $B$ and every neighborhood $U$ of $p^{-1}(b)$, there is a neighborhood $V$ of $p^{-1}(b)$ in $U$ such that the inclusion induced map $\tilde{H}_{k}(V) \rightarrow \tilde{H}_{k}(U)$ [resp. $\pi_{k}(V, e) \rightarrow \pi_{k}(U, e)$ ] is zero [for each base point $e$ in $V$ ]. The notation $u v^{k}$ [resp., $U V^{k}$ ] means $i$-uv [resp., $i-U V$ ] for all $i \leqslant k$. In the next section we define properties called $\pi_{k}$-movable and $H_{k}$-movable which generalize the " $U V$ " properties by allowing nonzero images. One of the fundamental theorems on $U V$ properties is the following Hurewicz-type theorem [L2, Theorem 4.2]. If $p: E \rightarrow B$ is a $U V^{k-1}$ mapping and a $k$-uv mapping where $k \geqslant 2$, then $p$ is a $U V^{k}$ mapping. The purpose of this note is to prove an analogous theorem for movable mappings.

THEOREM A. Let $p: E \rightarrow B$ be a mapping between locally compact separable ANR's. If $p$ is $U V^{1}, \pi_{i}$-movable for $i \leqslant k-1$, and $H_{k}$ - and $H_{k+1}$-movable where $k \geqslant 2$, then $p$ is $\pi_{k}$-movable.

Received by the editors December 12, 1978 and, in revised form, March 20, 1979.

AMS (MOS) subject classifications (1970). Primary 54C10; Secondary 54C55, 55C15, 55F65, 57A15.

Key words and phrases. Approximate fibration, $U V$ property.

${ }^{1}$ Research of both authors supported by N.S.F. contract. 
As an application, we give the following improvement of [CD2, Theorem 3.7] for $U V^{1}$-mappings, and a result on mappings between manifolds which generalizes [ $\mathbf{L}$, Theorem 5.4].

THEOREM B. If $p: E \rightarrow B$ is a $U V^{1}, \pi_{i}$-movable map for $i \leqslant k$ and each fiber of $p$ has fundamental dimension $\leqslant k$, then $f$ is an approximate fibration.

TheOREM C. Let $f: M^{m} \rightarrow N^{n}$ be a $U V^{1}$ mapping between manifolds. If $f$ is $\pi_{i}$-movable for all $i \leqslant k-1$ where $k \geqslant \max \{m-n+1,(m+1) / 2\}$, then $f$ is an approximate fibration.

We use the following terminology and notation in this paper. If $p: E \rightarrow B$ is a mapping, $b \in B$ or $U \subset B$, then the fiber $p^{-1}(b)$ is denoted by $F_{b}$ and $p^{-1}(U)$ is denoted by $\tilde{U}$. Our usual homology and cohomology groups are singular, with integral coefficient groups. If $\lambda$ is one of the usual homology, (cohomology) or homotopy functors, then $\check{\lambda}(X)$ denotes the inverse (direct) limit of $\lambda(U)$ as $U$ ranges over the neighborhoods of $X$. An absolute neighborhood retract for metric spaces is abbreviated to ANR. A manifold is assumed to be connected and boundaryless.

2. Movability and lifting properties. Suppose that $p: E \rightarrow B$ is a proper surjective map. We say that $p$ has the approximate homotopy lifting property $(A H L P)$ for $a$ space $X$ if for each commutative diagram

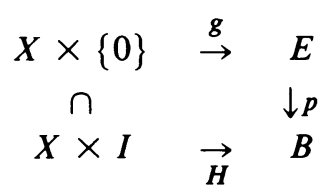

and open cover $\alpha$ of $B$, there is an extension $G: X \times I \rightarrow E$ of $g$ such that $p \circ G$ is $\alpha$-close to $H$. We say that such a $G$ is an $\alpha$-lift of $H$. If $p$ has the AHLP for all spaces $X, p$ is an approximate fibration.

In [CD2], it was shown that the AHLP for polyhedra can be detected by a homotopy regularity condition on fibers, which we called $k$-movability. In this paper, it will be convenient to define movability in a slightly more general setting.

Let $\Lambda_{0}$ be the collection of functors $\left\{\pi_{i}, H_{j} \mid i, j=0,1,2, \ldots\right\}$. If $\Lambda \subset \Lambda_{0}$, we say that $p$ is $\Lambda$-movable provided that given $b \in B$ and any neighborhood $U_{0}$ of $F_{b}$, there exist open sets $U$ and $V$ with $F_{b} \subset V \subset U \subset U_{0}$ such that for each $F_{c} \subset V$, each $\lambda \in \Lambda$ (and each base point in $F_{c}$ if relevant), the inclusion induced map sends $\check{\lambda}\left(F_{c}\right)$ isomorphically onto the image of $\lambda(V)$ in $\lambda(U)$. Given such $U, V$, we say that $\check{\lambda}\left(F_{c}\right)$ is realized as the image of $\lambda(V)$ in $\lambda(U)$.

Thus our earlier terminology, $k$-movable, is replaced in this paper by $\Lambda$-movable where $\Lambda=\left\{\pi_{i} \mid i \leqslant k\right\}$. Subject to this change the result from [CD2, Theorem 3.3] says that if $E$ and $B$ are ANR's and $f$ is $\Lambda$-movable for $\Lambda=\left\{\pi_{i} \mid i \leqslant k\right\}$, then $f$ has the AHLP for polyhedra of dimension $\leqslant k$.

We will need the following technical lemma. 
Lemma 2.1. Let $\Lambda_{1}$ and $\Lambda_{2}$ be subsets of $\Lambda_{0}$. If $p: E \rightarrow B$ is $\Lambda_{1}$-movable and $\Lambda_{2}$-movable, then $p$ is $\left(\Lambda_{1} \cup \Lambda_{2}\right)$-movable. Furthermore, if $B$ is a manifold, the open sets $U$ and $V$ may be chosen to be preimages of contractible sets.

Proof. Given $b \in B$ and $U_{0} \supset F_{b}$, choose open sets $U_{1}, V_{1}, U_{2}, V_{2}, U_{3}, V_{3}$ with $F_{b} \subset V_{3} \subset U_{3} \subset V_{2} \subset U_{2} \subset V_{1} \subset U_{1} \subset U_{0}$ such that for every $F_{c} \subset V_{3}, \lambda \in \Lambda_{1}$, $\check{\lambda}\left(F_{c}\right)$ is realized as the image of $\lambda\left(V_{3}\right)$ in $\lambda\left(U_{3}\right)$ and as the image of $\lambda\left(V_{1}\right)$ in $\lambda\left(U_{1}\right)$ and such that $\check{\mu}\left(F_{c}\right)$ is realized as the image of $\mu\left(V_{2}\right)$ in $\mu\left(U_{2}\right)$ for every $\mu \in \Lambda_{2}$. It is easy to check that if $U=U_{2}$ and $V=V_{3}, \check{\lambda}\left(F_{c}\right)$ is realized as the image of $\lambda(V)$ in $\lambda(U)$ for each $\lambda \in \Lambda_{1} \cup \Lambda_{2}$.

For the second conclusion, given $b \in B$ and $U_{0} \supset F_{b}$ choose open sets $V \subset V_{2}$ $\subset U_{2} \subset U \subset V_{1} \subset U_{1}$ such that $F_{b} \subset V, U_{1} \subset U_{0}, \check{\lambda}\left(F_{c}\right)$ is realized as the image of $\lambda\left(V_{i}\right)$ in $\lambda\left(U_{i}\right)$ for each $F_{c} \subset V_{i}(i=1,2)$, and $U$ and $V$ are preimages of contractible sets.

In the next section, it will be convenient to assume that $E$ and $B$ are $Q$-manifolds. A natural device is to replace $p$ by the map $p \times 1_{Q}: E \times Q \rightarrow B \times Q$ and appeal to Edwards' Theorem [E]. The reader can easily provide a proof for the following lemma.

LeMMA 2.2. If $p: E \rightarrow B$ is a proper map between $A N R$ 's, then for each of the properties $\mathrm{P}_{i}$ below, $p$ has $\mathrm{P}_{i}$ if and only if $p \times 1_{Q}$ has $\mathrm{P}_{i}$.

$\mathrm{P}_{1}$ : Being an approximate fibration.

$\mathrm{P}_{2}$ : Being $\lambda$-movable for some $\lambda \in \Lambda_{0}$.

$\mathrm{P}_{3}$ : Having the AHLP for a space $X$.

LemMa 2.3. Suppose that $p: E \rightarrow B$ is a map between $A N R$ 's and that $p$ has the $A H L P$ for polyhedra of dimension $\leqslant q$. If $V \subset U$ is a pair of open sets in $Y$, then $p_{*}$ : $\pi_{i}(\tilde{U}, \tilde{V}) \rightarrow \pi_{i}(U, V)$ is an isomorphism for $i \leqslant q$ and is epic for $i=q+1$.

The proof is a variation of a standard argument; see for example [S, Theorem 7.2.8], [L1, Corollary 2.4] and [L2, Lemma 1.2]. It uses [CD2, Lemma 1.2].

3. Proof of Theorem A. By Lemma 2.1 we may assume that both $E$ and $B$ are $Q$-manifolds. Thus, each point in $B$ has arbitrarily small contractible open neighborhoods.

Let us say that $p$ has property $i-D U V$ provided that for each $b \in B$ and each neighborhood $U_{0}$ of $F_{b}$, there are neighborhoods $V \subset U$ of $F_{b}$ in $U_{0}$ such that given any fiber $F_{c}$ in $V$ and any neighborhood $W_{0}$ of $F_{c}$ in $V$, there are neighborhoods $X \subset W$ of $F_{c}$ in $W_{0}$ such that the inclusion induced map $\nu_{\#}: \pi_{i}(V, X) \rightarrow$ $\pi_{i}(U, W)$ is the zero homomorphism for each base point. By Lemma 3.1 of [CD2], $p$ has property $i-D U V$ for $i \leqslant k-1$. We wish to prove $k-D U V$ and $(k+1)-D U V$.

Given $b \in B$ and a neighborhood $U_{0}$ of $F_{b}$, apply the hypotheses and Lemma 2.1 to choose $V \subset U$ satisfying the following properties.

(i) $\check{\pi}_{k-1} F_{c}$ is realized as the image of $\pi_{k-1} V$ in $\pi_{k-1} U$ for each $F_{c} \subset V$,

(ii) $\check{H}_{i} F_{c}$ is realized as the image of $H_{i} V$ in $H_{i} U$ for each $F_{c} \subset V$ and $i=k, k+$ 1 , and 
(iii) $U$ and $V$ are the preimages of contractible neighborhoods of $b$. Given a fiber $F_{c} \subset V$ and a neighborhood $W_{0}$ of $F_{c}$ in $V$, apply the hypotheses and the above lemmas again to choose $X \subset W$ satisfying the following properties:

(iv) $\check{\pi}_{k-1} F_{c}$ is realized as the image of $\pi_{k-1} X$ in $\pi_{k-1} U$,

(v) $\check{H}_{i} F_{c}$ is realized as the image $H_{i} X$ in $H_{i} W$, for $i=k, k+1$, and

(vi) $X$ and $W$ are preimages of contractible neighborhoods of $b$.

Consider the following commutative diagram.

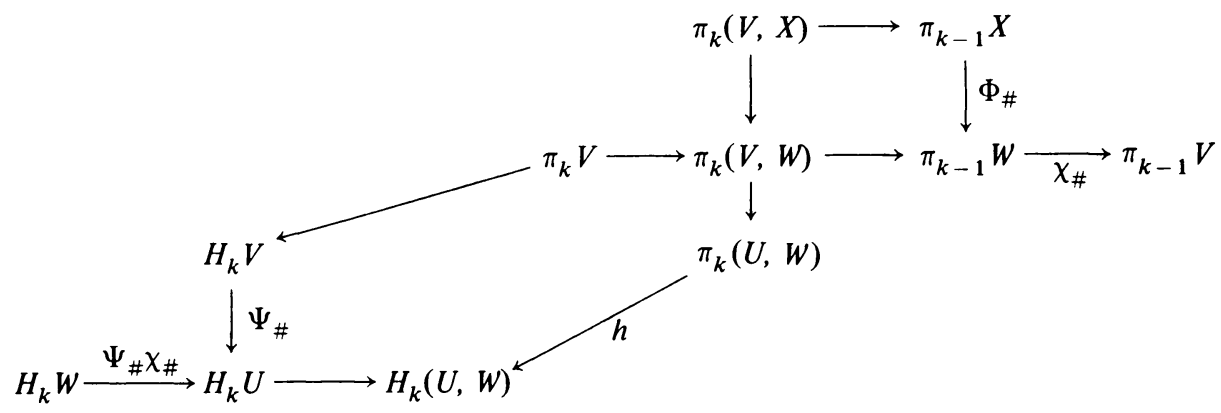

The horizontal rows are portions of exact sequences of pairs, the vertical arrows are inclusion-induced and the diagonal arrows are Hurewicz homomorphisms. By [L2, Lemma 5.1], (iii), and (vi), $U$ and $W$ are simply connected. Also by (iii) and (vi) and Lemma 2.3, $\pi_{i}(U, W)=0$ for $i \leqslant k-1$. Thus, by [S, p. 397] $h$ is an isomorphism. By (i) and (iv) $\chi_{\#} \lim \Phi_{\#}$ is monic, and by (ii) and (v) im $\Psi_{*} \chi_{*}=$ $\operatorname{im} \Psi_{*}$. It is now an easy "diagram chasing" argument to show that $\nu_{\#}$ is zero and, hence, we have $k-D U V$.

Consider next the following similar diagram.

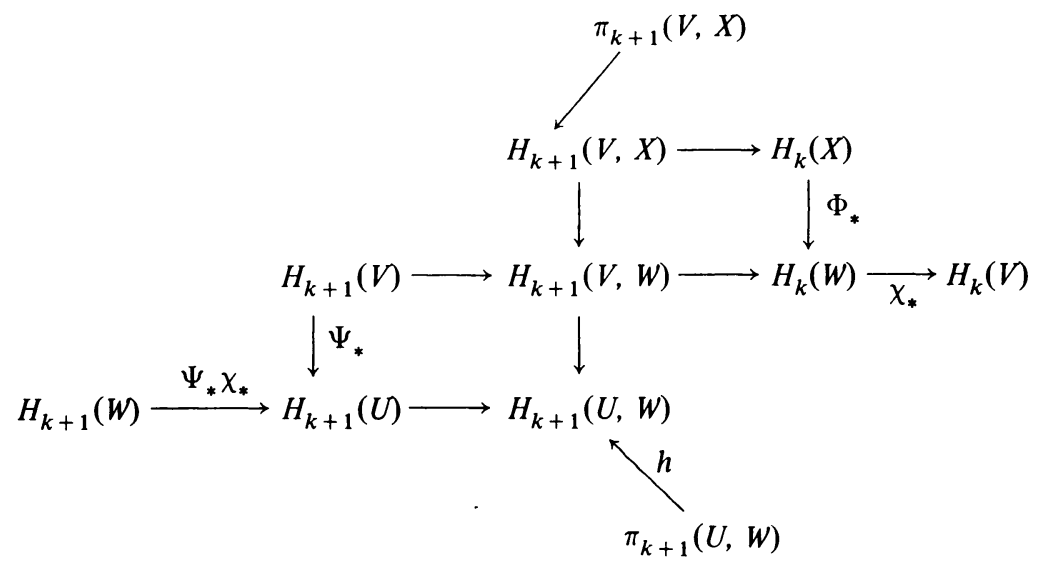

Since the proof of Theorem 3.3 of [CD2] really uses only $i-D U V$ (rather than $i$-movability as stated), we see that $p$ has AHLP for $I^{i}, i<i<k$. As above this implies that $\pi_{k}(U, W)=0$ and $h$ is an isomorphism. Also $\chi_{*} \lim \Phi_{*}$ is monic and $\operatorname{im} \Psi_{*}=\operatorname{im} \Psi_{*} \chi_{*}$ again. Hence $\nu_{\#}$ is zero and $(k+1)-D U V$ results. 
We now apply Theorem 3.3 of [CD2] again to see that $p$ has AHLP for $I^{i}$, $i<k+1$. Hence $p$ is a $\pi_{k}$-movable map by Proposition 3.5 of [CD2], so the proof is finished.

COROLlARY. Let $p: E \rightarrow B$ be a mapping between locally compact, separable metric $A N R$ 's. If $p$ is $U V^{1}$ and $H_{i}$-movable for all $i$, then $p$ is an approximate fibration.

When Theorem A is compared to Lacher's Hurewicz-type theorem for $U V$ properties [L2, Theorem 4.2], a discrepancy in the analogy is noticeable. There is no hypothesis in Lacher's theorem coresponding to the $H_{k+1}$-movable hypothesis in Theorem A. The reason for this difference is explained in the remark on page 51 of [CD2]. The extra hypothesis is necessary as the following example shows.

Let $f: S^{3} \rightarrow S^{2}$ be the Hopf fibration (a generator of $\pi_{3}\left(S^{2}\right) \cong Z$ ), and let $K$ be the complex obtained by attaching a 4-cell to the mapping cylinder $M_{f}$ along $S^{3}$ by the identity. It follows that

$$
\begin{aligned}
& \tilde{H}_{i}(K) \cong 0, \quad i \leqslant 1, \\
& H_{2}(K) \cong Z \cong \pi_{2}(K), \\
& H_{3}(K) \cong 0 \cong \pi_{3}(K), \quad \text { and } \\
& H_{4}(K) \cong Z .
\end{aligned}
$$

Let $\alpha: S^{2} \rightarrow K$ be a generator of $\pi_{2}(K)$ and let $M_{\alpha}=\left(S^{2} \times I \cup K\right) /\{(x, 1)=$ $f(x)\}$ be the mapping cylinder of $\alpha$. Define $p: M_{\alpha} \rightarrow I$ by a $p[(x, t)]=t, p(K)=1$. Then $p$ is a $\pi_{2}$-movable map which is $H_{3}$-movable and $1-U V$, but $p$ is not $\pi_{3}$-movable, since $\pi_{3}(K)=0, \pi_{3}\left(S^{2}\right) \neq 0$. Thus we cannot remove the assumption that $p$ be $H_{k+1}$-movable in Theorem A.

\section{Proofs of the applications.}

Proof of Theorem B. Since $F d\left(F_{b}\right) \leqslant k, \check{H}^{i}\left(F_{b}\right) \cong 0$ for $i \geqslant k+1$ and each $b \in B$. It follows from [L2, Theorem 3.1] that $p$ is an $i-u v(Z)$ map for $i>k+1$. Hence $p$ is $H_{i}$-movable for $i \geqslant k+1$. By Theorem A,p is $\pi_{i}$-movable for all $i$, so $p$ is an approximate fibration by [CD2, Corollary 3.4].

LEMMA 4.1. If $f: M^{m} \rightarrow N^{n}$ is a $U V^{1},\left\{\pi_{i} \mid i \leqslant k-1\right\}$-movable mapping between manifolds, then for each $y \in N, \check{H}^{j}\left(F_{y}\right)=0$ for $j \geqslant \max \{m-k+1, m-n+1\}$.

Proof. For $n=0,1$, the result is contained in [LM, Theorem 1.3]. For $n \geqslant 2$, take $y \in N$ and $j \geqslant \max \{m-k+1, m-n+1\}$. If $U$ is a Euclidean neighborhood of $y$, then $U$ is simply connected and $\pi_{m-j}(U, U-y)=0$. By Lemma 2.3, $\pi_{m-j}\left(\tilde{U}, \tilde{U}-F_{y}\right)=0$. The relative Hurewicz theorem [S, p. 397] yields $H_{m-j}(\tilde{U}, \tilde{U}$ $\left.-F_{y}\right)=0$. Since $U$ is simply connected and $f$ is $U V^{1}, \tilde{U}$ is simply connected [L2, Lemma 5.1] and thus orientable [S, p. 294]. Therefore duality [S, p. 296] can be applied to give $\check{H}^{j}\left(F_{y}\right)=0$.

Proof of Theorem C. Let $y \in N$. By the above lemma $\check{H}^{j}\left(F_{y}\right)=0$ for all $j \geqslant \max \{m-k+1, m-n+1\}$. Since $k \geqslant m-n+1$ and $k \geqslant(m+1) / 2$, $\max \{m-k+1, m-n+1\} \leqslant k$. Hence $\check{H}^{j}\left(F_{y}\right)=0$ for all $j \geqslant k$. By [L, Theorem 3.1], $f$ is a $j$-uv map for all $j \geqslant k$; and by Theorem A, $f$ is $\left\{\pi_{i}\right\}_{i=1}^{\infty}$-movable. Hence $f$ is an approximate fibration [CD2, Corollary 3.4]. 


\section{REFERENCES}

[CD1] D. Coram and P. Duvall, Approximate fibrations, Rocky Mountain J. Math. 7 (1977), 275-288. [CD2] _ Approximate fibrations and a movabilitity condition for maps, Pacific J. Math. 72 (1977), 41-56.

[E] R. D. Edwards, A locally compact ANR is a Hilbert cube manifold factor (to appear).

[L1] R. C. Lacher, Cell-like mappings. I, Pacific J. Math. 30 (1969), 717-731.

[L2] R. C. Lacher, Cellularity criteria for maps, Michigan Math. J. 17 (1970), 385-396.

[LM] R. C. Lacher and D. R. McMillan, Partially acyclic mappings between manifolds, Amer. J. Math. 94 (1972), 246-266.

[S] E. H. Spanier, Algebraic topology, McGraw-Hill, New York, 1966.

Department of Mathematics, OkLahoma State University, Stmlwater, OrLahoma 74074 